\title{
La elaboración de la tesina como la última cuesta en la graduación: Sus dificultades y el rol de tutoría
}

\author{
Thesis Preparation as the Last Step to Graduation: \\ Difficulties and Thesis Supervision
}

\author{
Teresita Alcia Izura' \\ Facultad de Educación Elemental y Especial \\ Universidad Nacional de Cuyo \\ Argentina \\ pupuiz@yahoo.com.ar \\ Laura María Torres ${ }^{2}$ \\ Facultad de Educación Elemental y Especial \\ Universidad Nacional de Cuyo \\ Argentina \\ Itorres@mendoza-conicet.gob.ar
}

1 Licenciada en Ciencias Políticas y Administración Pública por la Universidad Nacional de Cuyo. Doctora por la Universidad de Jaén, España, en Humanidades y Ciencias Sociales. Magister en Ciencias Sociales por la Facultad Latinoamericana de Ciencias Sociales, cuenta, además, con diplomaturas y especializaciones afines a metodología de investigación y estadística. Investigadora y docente efectiva en las cátedras de Metodología y técnicas de investigación cuantitativas y cualitativas y en la cátedra de Estadística y Demografía, Facultad de Ciencias Políticas y Sociales y en la Facultad de Educación Elemental y Especial de la Universidad Nacional de Cuyo y en otras universidades. Participa de numerosos eventos científicos y ha recibido premios, subsidios y becas.

Dirección Profesional: Facultad de Ciencias Políticas y Sociales, Parque General San Martín, Ciudad Universitaria, Ciudad, Mendoza. Teléfono: 0054261 4135008, interno 2043.

2 Investigadora adjunta del Consejo Nacional de Investigaciones Científicas y Tecnológicas (CONICET), con lugar de trabajo en el Instituto Argentino de Investigaciones de las Zonas Áridas (IADIZA - CCT CONICET Mendoza). Se desempeña, además, como docente-investigadora de la Facultad de Educación Elemental y Especial, dependiente de la Universidad Nacional de Cuyo. Licenciada en Trabajo Social por la Universidad Nacional de Cuyo. Magíster en Antropología por la Universidad Nacional de Córdoba. Doctora en Antropología Social por la Universidad de Sevilla. En el seno de la Facultad de Educación Elemental y Especial (UNCuyo) se desempeña al interior del Departamento de Metodología de la Investigación y desarrolla una línea de investigación interesada por el estudio de los procesos de elaboración de los trabajos de tesis. 
URL: http://www.una.ac.cr/educare

CORREO: educare@una.cr

\author{
Fabiana María Böhm Carrer ${ }^{3}$ \\ Facultad de Educación Elemental y Especial \\ Universidad Nacional de Cuyo \\ Mendoza, Argentina \\ bohmcarrer@yahoo.com.ar \\ Sergio Edgardo Terrera ${ }^{4}$ \\ Facultad de Educación Elemental y Especial \\ Universidad Nacional de Cuyo \\ Mendoza, Argentina \\ sterrera@yahoo.com \\ Adrián Edgardo Lucero 5 \\ Facultad de Educación Elemental y Especial \\ Universidad Nacional de Cuyo \\ Mendoza, Argentina \\ wine_xxi@yahoo.com.ar
}

Recibido 06 de noviembre de 2012 • Corregido 06 de marzo de 2013 • Aceptado 13 de marzo de 2013

3 Licenciada en Sociología por la Universidad Nacional de Cuyo - UNC- , Mendoza, Argentina y ha terminado de cursar, en el año 2010, la Maestría en Criminología en la Universidad del Aconcagua - UDA - de Mendoza. Actualmente se desempeña como docente en la Carrera de Trabajo Social de la Facultad de Ciencias Políticas y Sociales de la U.N.C, y en la Carrera de Niñez, Adolescencia y Familia de la Facultad de Psicología de la U.D.A.

Posee antecedentes como integrante de grupos de investigación vinculados a las temáticas del género y el trabajo, aspectos de la educación superior y problemática actuales de la criminología. Directora y co directora de numerosos proyecto de investigación. Ha publicado artículos y capítulos de libros en torno a la metodología de investigación y su relación con el uso de diversos software.

4 Licenciado en Documentación y Gestión de la Información. Egresado de la Facultad de Educación Elemental y Especial, UNCuyo, distinguido con las medallas "Mejor promedio Tecnicatura en Documentación" (2007) y"Mejor egresado de los ciclos de Licenciaturas" (2011), donde se desempeña como profesor invitado a cargo de la asignatura "Introducción a las Ciencias de la Información". Acredita posgrados de formación continua en su campo de formación. En el área de investigación participa de numerosos equipos de investigación en la FEE y Especial de la UNCuyo. Asimismo, ha presentado numerosas ponencias en encuentros científicos y ha recibido numerosos premios, es evaluador especialista en la Dirección General de Escuelas, para proyectos de capacitación a los Bibliotecarios Escolares y desde el 2001 es el responsable efectivo de la Biblioteca del Agua, del Departamento General de Irrigación.

5 Licenciado en Documentación y Gestión de la Información. Técnico Universitario en Documentación, por la Universidad Nacional de Cuyo. Actualmente cursa el Posgrado en Especialización en Docencia Nivel Superior, Universidad J.A. Maza, Mendoza.

Actividades profesionales actuales: Instituto Nacional de Vitivinicultura, Biblioteca Especializada Universidad J.A. Maza, Biblioteca Universitaria. Ha presentado numerosas ponencias y obtenido premios y acredita múltiples cursos realizados en torno al uso de biblioteca electrónica, biblioteca digital, bibliotecas populares, públicas y escolares. Manejo de software para análisis de datos cualitativos: Atlas.Ti, Gestión de calidad, Interpretación e implementación de la NORMA ISO 9001, Gestión de la información para el desarrollo integrado. Ha participado de programas de entrenamiento laboral en organización, grupos y liderazgo e introducción a la Ley de Administración y técnicas de control en el sector público, en INAP. 
Resumen. Este es un artículo científico derivado de un trabajo de investigación llevado a cabo en la Facultad de Educación Elemental y Especial de la Universidad Nacional de Cuyo, cita en Mendoza, Argentina, en la que se dictan ocho ciclos de licenciaturas que requieren, para su titulación, la realización de una tesina. Si bien el objetivo del estudio en que se enmarca este escrito es indagar acerca de los trayectos de tesina, tanto desde el relato de los egresados como de los directores, en esta instancia, se pondrá especial énfasis en aquellos hallazgos obtenidos, vinculados con el rol del director o tutor, los esquemas de responsabilidades que se le asignan y las verdaderas limitaciones de su tarea. De ello surgen una serie de interrogantes e implicaciones respecto al papel que a la institución le cabe al respecto. A modo de ilustración de la diversidad de emergentes que se registran en el ámbito de la observabilidad, se desplegará parte de los resultados de una investigación amparada en la estrategia metodológica cuantitativa, que pone al desnudo y en variables el entramado de dificultades que se presentan frente a la terminalidad de diversos ciclos de estudio. Se trabajó con un diseño descriptivo. Conformaron las unidades de observación los estudiantes de las carreras pertenecientes a la institución en cuestión. El trabajo con datos secundarios suministrados por la misma institución y técnicas estadísticas, permitió sistematizar la información obtenida para su análisis y posterior decantación en resultados pertinentes. Los hallazgos permiten establecer algunas implicaciones vinculadas al rol de los tutores, al significado de la elaboración de una tesina, a las brechas temporales y a los vacíos institucionales en torno a estas problemáticas. Todo esto invita a revisar el compromiso institucional, en cuanto a los docentes que se desempeñan como tutores y a los estudiantes en la particular etapa de tránsito del proceso de elaboración de la tesina.

Palabras claves. Terminalidad, tesina, tutor, formación de grado, estudio de caso.

Abstract. This paper is the result of a research project conducted in the Facultad de Educación Elemental y Especial of the Universidad Nacional de Cuyo, Mendoza, Argentina, where we have eight cycles of licenciaturas, which require a dissertation as a final project to graduate. Although the purpose of this study is to know the stages in the preparation of a dissertation -from the point of view of individuals graduated and thesis supervisors-, we will pay special attention to the role of the thesis supervisor, the scheme of his/her responsibilities and the real limitations of his/her work. Many questions arise concerning the role of the institutions on this matter. As an example, this paper provides the results obtained from a research based on a quantitative methodological strategy, which shows the difficulties observed at the end of different study cycles. A descriptive design was used. The observation units of this study were the students from the participant institution. The secondary data provided by the same institution and the statistical techniques used facilitated the systematization of the information obtained for its analysis and subsequent classification of relevant results. The findings enable us to see some implications related to the role of thesis supervisors, the significance of preparing a dissertation, and the institutional temporary gaps on this matter. In conclusion, this paper is an invitation to review the institutional commitment in terms of the teachers working as thesis supervisors and the students going through the thesis preparation.

Keywords. End, thesis, tutor, formation, case study. 
URL: http://www.una.ac.cr/educare

CORREO: educare@una.cr

Se asiste, en la actualidad, a grandes modificaciones en los paradigmas vinculados a la educación y a la mediación de los aprendizajes. Todo mutó del paradigma de enseñanza al del aprendizaje, y la era del conocimiento acuña su impronta en los diversos niveles educativos de la sociedad.

También aparece una instancia superadora del ciclo de base que constituye el nivel de grado, ya que la multiplicidad de posgrados ofrecidos y el creciente número de titulaciones susceptibles de adquirirse en el trayecto de formación académica hacen del primer nivel mencionado un punto de partida para lo que parece será la formación especializada en diversos tópicos disciplinares.

Frente a estos nuevos escenarios, donde la oferta de formación desafía el tiempo disponible de los profesionales de cada disciplina y donde la pertenencia al mundo académico exige la constante profesionalización, resulta, cuando menos atractivo, conocer algunas particularidades vinculadas a demoras elocuentes en la terminación del ciclo de formación de grado.

Reiteradas observaciones realizadas en ámbitos académicos y, especialmente, en la institución objeto de este estudio, denotan vacancia vinculada al tratamiento de los inconvenientes en los procesos de finalización de las tesis o tesinas, según sea el nivel del cual se trate.

Para esta comunicación ${ }^{6}$ se ha operativizado la clase textual tesina como adecuada, ya que alude a la terminalidad de las carreras de licenciatura, en un tipo de trabajo donde debe darse cuenta de la experticia en procesos de investigación y presentación de resultados.

La institución seleccionada para visualizar el fenómeno en cuestión es la Facultad de Educación Elemental y Especial de la Universidad Nacional de Cuyo (Mendoza, Argentina), cuyo “(...) ámbito de competencia comprende la formación universitaria de Profesores y Licenciados para los niveles inicial, primero y segundo ciclos de Educación General Básica, la educación de sujetos con necesidades especiales y la educación de adultos" (UNCUYO. FEEyE, párr. 3).

Esta Facultad cuenta con una modalidad que desdobla las titulaciones de grado en dos niveles: el profesorado y la licenciatura. Ofrece ocho licenciaturas, cada una de ellas de dos años de duración y a las que pueden acceder los profesores egresados de la Facultad y otros profesionales del ámbito provincial, nacional e internacional que acrediten una titulación de técnico universitario.

Las licenciaturas que se desarrollan en el ámbito de la Facultad de Educación Elemental y Especial son:

- Licenciatura en Gestión de la Educación Especial

- Licenciatura en Gestión Institucional y Curricular

- Licenciatura en Educación Inicial

- Licenciatura en Creatividad Educativa

6 Avances preliminares de este trabajo han sido presentados en el III Encuentro Latinoamericano de Metodología de las Ciencias Sociales (ELMeCS), "Métodos alternativos o críticos de la investigación en ciencias sociales" Construcción de opciones metodológicas para las ciencias sociales contemporáneas, Manizales (Colombia), 30-31 de agosto y 1 de septiembre de 2012. 
- Licenciatura en Educación Ambiental

- Licenciatura en Fonoaudiología

- Licenciatura en Psicomotricidad Educativa

- Licenciatura en Documentación y Gestión de la Información

Las características particulares de la unidad educativa seleccionada como estudio de caso han permitido profundizar los procesos de comparación constante y han brindado, además, amplias oportunidades para indagar dimensiones de la realidad relativamente poco exploradas en la bibliografía a disposición.

Desde la información en cifras obtenida y procesada en la investigación que encuadra este escrito, se ha podido avanzar en evidencias que permiten sostener una hipótesis central:

La terminación de las tesinas en las diversas carreras de licenciatura de la Facultad de Educación Elemental y Especial presenta importantes brechas temporales, años de desgranamiento entre la culminación del tramo de aprobación de la última materia y la finalización de dicha tesina.

Así pues, la estrategia metodológica prevista para la primera parte del estudio es de corte cuantitativo y, para poder dar cuenta de la hipótesis, se trabajará con datos secundarios, proporcionados por Secretaria Académica de la mencionada institución.

Luego de esta suerte de diagnóstico descriptivo, el énfasis particular será depositado sobre el rol del tutor en cuanto a la realización de las tesinas. Y esto es así por cuanto la investigación madre de este trabajo, en una segunda etapa de corte cualitativo, arrojó un conjunto de resultados que constituyen hallazgos novedosos y resultan interesantes aportes acerca del objeto de estudio.

Para este escrito, se recortan los resultados entonces obtenidos y se focaliza exclusivamente en lo que al rol del tutor o director de tesina concierne. Se busca generar un producto acotado y autónomo que dé cuenta del estado de esta particular problemática.

\section{Las dificultades evidenciadas en cifras}

A continuación, se pretende sintetizar en tablas y figuras, algunas de las variables más significativas vinculadas a las dificultades en la terminación de los estudios de grado para las carreras ya mencionadas de la Facultad de Educación Elemental y Especial.

Para este trabajo, se extrajo parte de la información de base de la investigación que le da origen. Concretamente se han seleccionado los años 2010 y 2011 respecto la cantidad de personas que rindieron su última materia y las que presentaron su tesina, en cada una de las ocho licenciaturas dictadas. 
URL: http://www.una.ac.cr/educare

CORREO: educare@una.cr

En la primera tabla que se presenta es posible analizar un dato potente, constituido por el rango que se establece entre el año en que se rindió la última materia y el año en que se egresó, es decir, el propio a la presentación y defensa de la tesina correspondiente.

Solo se vislumbra, en una de las ocho licenciaturas mencionadas, el caso de tres personas egresadas que han concluido, en el mismo año, los compromisos correspondientes al cursado y la aprobación de materias con las obligaciones orientadas a la elaboración y defensa de la tesina.

Sin embargo, realizando un análisis de los años de demora entre los dos momentos establecidos, se aprecia que en el resto de las licenciaturas, el recorrido va entre los 3 y los 9 años, donde la demora modal corresponde a los 6 años y el promedio de años de demora a 3,87.

Para los ciclos de licenciatura mencionados, se hallan involucradas 16 personas que, atendiendo al año en que rindieron su última materia, consignan diversos periodos de desgranamiento.

Tabla 1

Tabla integrada entre personas que rindieron su última materia según año y licenciatura y fecha de presentación de tesina para los años seleccionados. Consigna la mayor brecha entre años. Años 2010 y 2011

\begin{tabular}{lcccc}
\hline Licenciatura en & $\begin{array}{c}\text { Total } \\
\text { personas }\end{array}$ & $\begin{array}{c}\text { Año de última } \\
\text { materia }\end{array}$ & $\begin{array}{c}\text { Año de } \\
\text { egreso }\end{array}$ & $\begin{array}{c}\text { Brecha en } \\
\text { años }\end{array}$ \\
\hline Creatividad & 2 & 2005 & 2010 & 5 \\
Documentación y gestión de la información & 1 & 2008 & 2011 & 3 \\
Educación ambiental & 4 & 2007 & 2011 & 4 \\
Educación inicial & 1 & 2004 & 2010 & 6 \\
Fonoaudiología & 3 & 2010 & 2010 & 0 \\
Educación especial & 2 & 2003 & 2010 & 7 \\
Gestión & 2 & 2004 & 2010 & 6 \\
Psicomotricidad & 1 & 2002 & 2011 & 9 \\
\hline
\end{tabular}

Nota: Elaboración propia sobre la base de información proporcionada por Oficina de Secretaria Académica (UNCUYO, FEEyE, 2011).

Así entonces, el mayor desgranamiento lo evidencia una persona que corresponde a la carrera de Licenciatura en Psicomotricidad y que espació nueve años la presentación de su trabajo de tesina en referencia a la fecha de aprobación de la última materia.

La figura 1 permite ver la frecuencia absoluta de estudiantes, por carrera y que a la vez forman parte de quienes participan de las brechas más significativas entre la aprobación de la última materia y la presentación de la tesis, lo cual tuvo lugar en los años 2010 o 2011. 
URL: http://www.una.ac.cr/educare

A partir de la observación de la información es posible visualizar que la mayor frecuencia corresponde a la carrera de Licenciatura en Educación Ambiental, cuatro individuos (en valores absolutos), porcentualmente un $25 \%$ del total de personas egresadas de la institución en estudio.

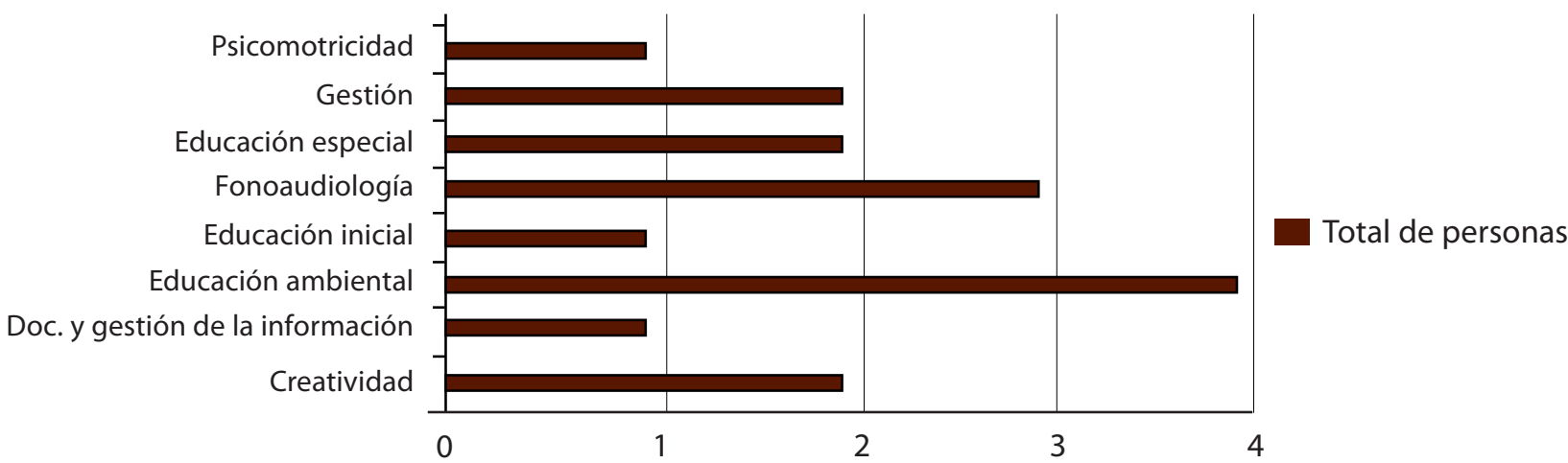

Figura 1. Total de personas por licenciatura partícipes de las mayores brechas

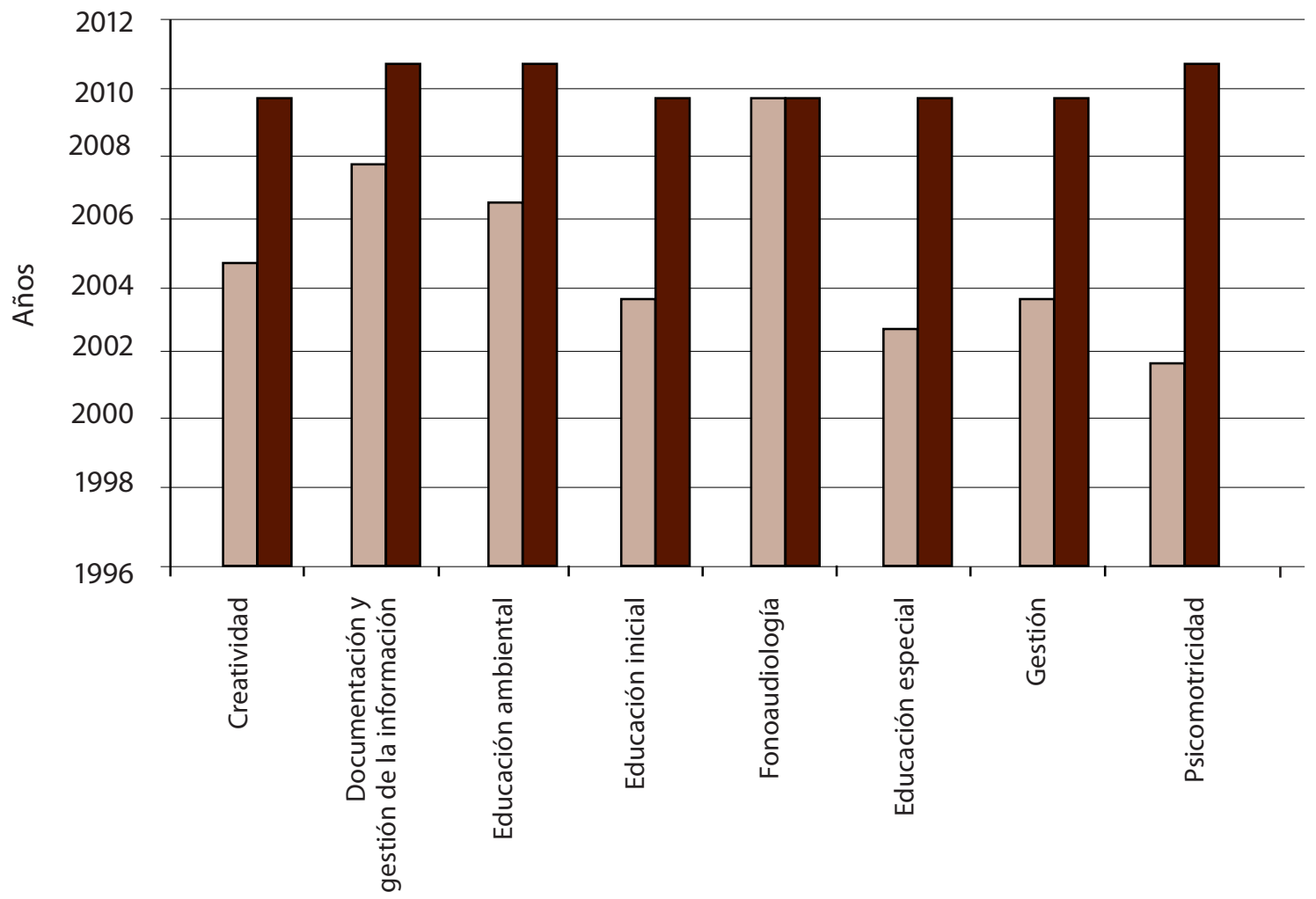

Licenciaturas

Figura 2. Año en que rindió última materia y año en que egresó 
URL: http://www.una.ac.cr/educare

CORREO: educare@una.cr

La figura precedente muestra una clara comparación entre los años en que se rindió la última materia y los años en que se egresó. Esta evidencia gráfica permite ver situaciones polares, ya que la Licenciatura en Fonoaudiología presenta barras apareadas paralelas e iguales, es decir, coinciden en el mismo año la aprobación de la última materia y la presentación y defensa de la tesina para las personas respectivas.

En tanto que, con una situación muy diferente, se visualiza a la Licenciatura en Psicomotricidad. Esta presenta la mayor distancia y diferencia entre el año de aprobación de la última materia y el año correspondiente a la presentación y defensa de la tesis. Mientras que en el primer caso todo ocurrió en el año 2010, en el segundo caso ocurrió entre los años 2002 y el 2011.

La figura de sectores a continuación, constituye información alternativa a la que se ha presentado precedentemente, pero ofrece otra manera, posiblemente más elocuente, de observar la información correspondiente a la brecha, en años, entre los dos momentos significativos seleccionados.

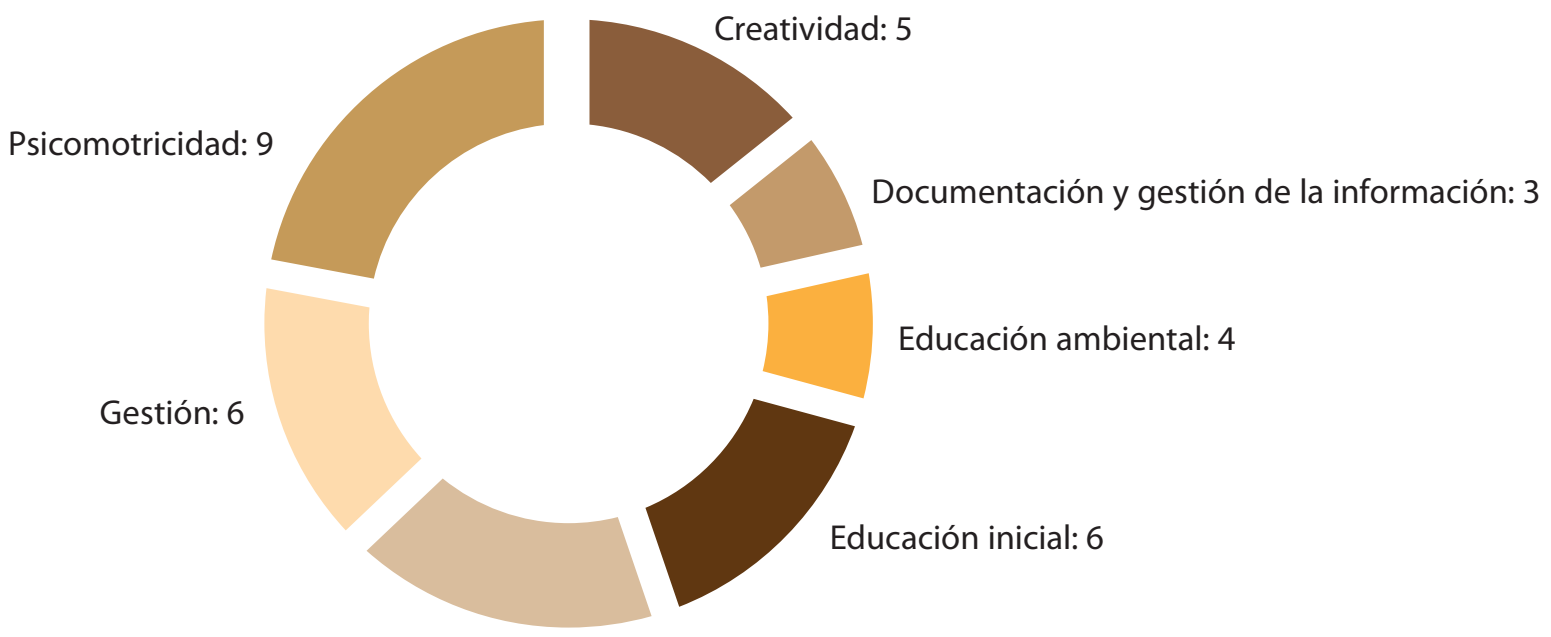

Educación especial: 7

Figura 3. Brecha en años entre fecha de última materia y presentación de tesis por licenciatura

Otro aspecto interesante que surgió, al analizar estos largos recorridos hacia la culminación de las carreras, se vinculó con las calificaciones obtenidas en promedio, por los estudiantes en sus tesinas.

Es evidente que las calificaciones son iguales o superiores a 9, lo que, desde esta perspectiva, permite afirmar que el rendimiento en esta última instancia ha sido considerablemente alto.

No obstante, se puedemencionarque las menores calificaciones en promedio corresponden a la Licenciatura en Documentación y Gestión de la Información, 9, y las calificaciones más elevadas corresponden a la Licenciatura en Educación Inicial y Fonoaudiología, 10. 
Tabla 2

Calificaciones promedio de tesis por licenciatura para el año 2010 y 2011

\begin{tabular}{lc}
\hline Licenciatura en & Calificación promedio \\
\hline Creatividad & 9,68 \\
Documentación y gestión de la información & 9, \\
Educación ambiental & 9,53 \\
Educación inicial & 10, \\
Fonoaudiología & 10, \\
Educación especial & 9,29 \\
Gestión & 9,41 \\
Psicomotricidad & 9,08 \\
\hline
\end{tabular}

Nota: Elaboración propia sobre la base de información proporcionada por Oficina de Secretaria Académica. (UNCUYO, FEEyE, 2011)

Si se realiza una mirada global de todas las licenciaturas en relación con la calificación promedio de sus tesis, las que consignan mayores y mejores notas son la Licenciatura en Educación Inicial y en Fonoaudiología.

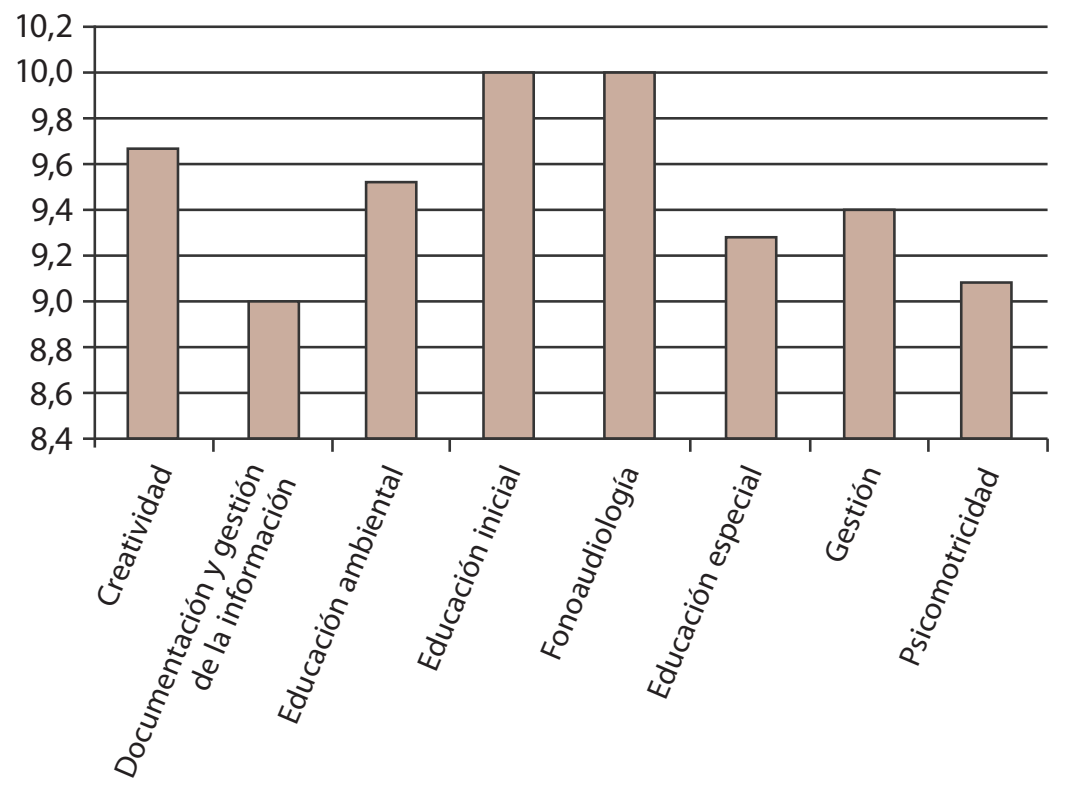

Figura 4. Calificación promedio de tesis por licenciatura 2010 y 2011 
URL: http://www.una.ac.cr/educare

CORREO: educare@una.cr

A continuación se presentan las tablas 3 y 4, que trabajan con las personas egresadas, entendidas como aquellos alumnos alumnas que rindieron la última materia, según frecuencias absolutas por carrera y para los dos años que se han seleccionado, esto es 2010 y 2011, diferenciados por sexo.

Tabla 3

Cantidad de mujeres egresadas por licenciatura y por año

\begin{tabular}{lccc}
\hline Licenciatura en & Cantidad mujeres & 2010 & 2011 \\
\hline Creatividad & 15 & 13 & 2 \\
Documentación y gestión de la información & 5 & 3 & 2 \\
Educación Ambiental & 14 & 6 & 8 \\
Educación Inicial & 2 & 2 & - \\
Fonoaudiología & 3 & 3 & 3 \\
Educación Especial & 18 & 15 & 21 \\
Gestión & 59 & 38 & 14 \\
Psicomotricidad & 38 & 24 & 50 \\
\hline Totales & 154 & 104 & \\
\hline
\end{tabular}

Nota: Elaboración propia sobre la base de información proporcionada por Oficina de Secretaria Académica. (UNCUYO, FEEyE, 2011).

Tabla 4

Cantidad de varones egresados por licenciatura y por año

\begin{tabular}{lccc}
\hline Licenciatura en & Cantidad varones & 2010 & 2011 \\
\hline Creatividad & 2 & 1 & 1 \\
Documentación y gestión de la información & 6 & 5 & 1 \\
Educación ambiental & 2 & 2 & - \\
Educación inicial & 0 & - & - \\
Fonoaudiología & 0 & - & - \\
Educación especial & 0 & - & 1 \\
Gestión & 4 & 3 & - \\
Psicomotricidad & 0 & - & 3 \\
\hline Totales & 14 & 11 & \\
\hline
\end{tabular}

Nota: Elaboración propia sobre la base de información proporcionada por Oficina de Secretaria Académica. (UNCUYO, FEEyE, 2011). 
Las dos tablas precedentes permiten observar que es ostensiblemente mayor el número de mujeres abocadas al estudio de estas licenciaturas sobre los varones; no obstante, en algunas de ellas se consigna la presencia masculina, por ejemplo, en Licenciatura en Creatividad, en Documentación y Gestión de la Información, en la Licenciatura en Educación Ambiental y en la de Gestión.

Tabla 5

Cantidad de personas egresadas por licenciatura y por año

\begin{tabular}{lccc}
\hline Licenciatura en & Egresadas en 2010 & Egresadas en 2011 & Totales \\
\hline Creatividad & 14 & 3 & 17 \\
Documentación y gestión de la información & 8 & 3 & 11 \\
Educación ambiental & 8 & 8 & 16 \\
Educación inicial & 2 & 0 & 2 \\
Fonoaudiología & 3 & 0 & 3 \\
Educación especial & 15 & 3 & 18 \\
Gestión & 41 & 22 & 63 \\
Psicomotricidad & 24 & 14 & 38 \\
\hline Totales & 115 & 53 & 168 \\
\hline
\end{tabular}

Nota: Elaboración propia sobre la base de información proporcionada por Oficina de Secretaria Académica. (UNCUYO, FEEyE, 2011).

La tabla 5 presenta, integrados, los valores correspondientes a varones, mujeres, carreras y los dos años que se han tomado como referencia, con sus respectivos totales.

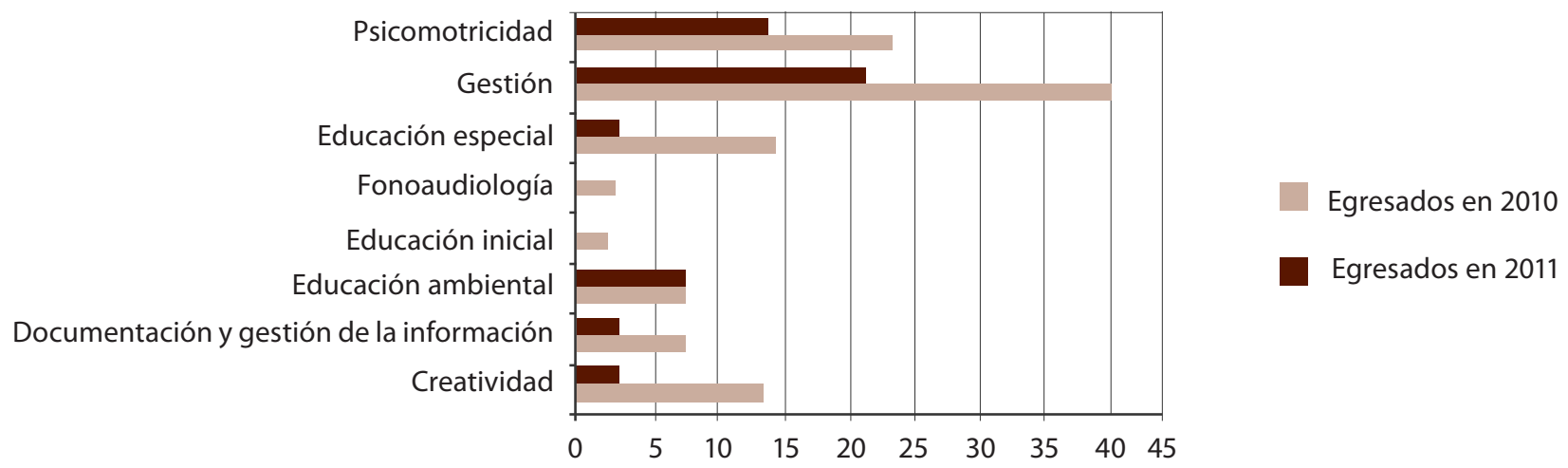

Figura 5. Total de personas egresadas en los años 2010 y 2011 por licenciatura 
URL: http://www.una.ac.cr/educare

CORREO: educare@una.cr

En síntesis, se puede colegir, del tratamiento de estos datos, la existencia de una demora sustantiva en los trayectos de estudiantes de las licenciaturas. Esta excede, por mucho, en años, los tiempos previstos en los currículos para este tipo de formación. Esta situación se destaca al trabajar con los años en que el alumnado rindió su última materia y, los años en que dieron por concluida su tesina.

Lamentablemente, no se ha podido seguir la suerte de desgranamiento que se produce año tras año, sino un trayecto entre los dos momentos extremos. Se advierte, del mismo modo, que el desgranamiento de los estudiantes se concentra en la mayoría de las carreras, aunque no en todas, ya que existen excepciones como la Licenciatura en Fonoaudiología y Educación Inicial, donde entre la fecha de presentación de la última materia y la posterior presentación de la tesinas se verifican años de diferencia y, en ciertos casos, estos años exceden el tiempo que les ha llevado a los estudiantes rendir todas las materias correspondientes.

Si el análisis se centra en la cantidad de personas egresadas en los dos años analizados, es posible observar que el $68,45 \%$ del grupo presentó su tesina en 2010 y con eso concluyó sus estudios de grado; en tanto que el 38,55 \% lo hizo en el año 2011.

Asimismo, al interior de las carreras trabajadas, la Licenciatura en Gestión evidencia los mayores porcentajes de egreso para ambos años, con el 35,6\% en el año 2010 y el 45,5\% en el 2011.

La observación generalizada permite aventurar algunos aspectos que pueden incidir, causalmente, en la situación de dilación que se evidencia. Por ejemplo, el hecho de que los ciclos de licenciatura tengan una organización posterior a los ciclos de profesorado hace que mucha gente que asiste a los primeros deba, a su vez, trabajar, lo que podría, en algún caso, justificar las demoras. En el mismo eje, el sentido de responsabilidad cambia y las personas quieren hacer procesos de formación más serios y concienzudos, lo que también puede contribuir a demandar más tiempo. Muchas de estas personas han perdido ritmo de estudio y, para el caso de la finalización de la carrera, la soledad de la elaboración de la tesis las paraliza. Además, y como se menciona posteriormente, hay algunos aspectos ligados de manera directa a la institución, como la falta de disponibilidad de directores o tutores, tanto de contenidos teóricos como metodológicos. En el mismo sentido, la falta de un seguimiento institucional frente al desgranamiento o de un programa abocado a acompañar la última etapa de elaboración de la tesina puede ser otro de los tópicos presentes.

\section{Tesinas y tutores}

Frente a la suerte de diagnóstico que el trabajo y sistematización de datos cuantitativos arrojó, en la primera fase de la investigación realizada -y que somera, pero acabadamente se ha presentado hasta aquí- se llevaron a cabo entrevistas en profundidad a los actores involucrados, es decir, egresados y egresadas recientes y a personal que fungió en funciones de dirección de tesinas, a fin de conocer, en sus propios relatos, acerca de las dificultades que los 
datos empíricos sugerían y que la revisión teórica avalaba. De esta indagación realizada surge la apreciación acerca de la gravitante importancia que reviste, en el proceso de tesina, el rol del director o directora.

Existe, en el imaginario, la idea de altos niveles de dificultad para la terminación de las tesinas; sin embargo, también hay evidencias más concretas. Ulrich y Smallwood reportan, en Rosas (2006), que las cifras indican que el abandono de los estudios doctorales en Estados Unidos se encuentra alrededor de 40 a 50\%, con un promedio de permanencia de 6 a 7 años.

Frente a parte de esa realidad, es interesante retomar y profundizar el conocimiento del fenómeno, tal lo hecho en la investigación referida, a cargo de los autores del presente escrito

Una buena apreciación sobre el trabajo de elaboración de una tesina se sintetiza en el aporte de Támola (2005) cuando sostiene que “(...) de todas formas, el esfuerzo por sistematizar los rasgos característicos de las Tesinas de licenciatura es ciertamente válido, porque resulta un intento por descubrir, a los que se están iniciando en el proceso de composición, el camino de textualización transitado por los que ya han ganado con creces su título" (p. 235).

Por su parte, Borsinger (2005) comenta que "(...) la tesis representa el ordenamiento del conocimiento obtenido durante la elaboración de un largo trabajo de acumulación, análisis y desarrollo de conocimiento" (p. 267).

A estas alturas resulta evidente la relevancia y complejidad que implica la elaboración de una tesis -o tesina para este caso particular-, por lo que es importante profundizar otras contribuciones autorizadas.

Los especialistas aportan conocimiento en relación con numerosos casos detectados en el marco de diversas investigaciones, e incluso generado en trabajos tales como el realizado por Valarino (1997), una suerte de taxonomía donde se establecen conceptos nominales o clasificatorios para trabajar con los casos que presentan dificultades con el trabajo final de tesina. Así, entonces, se habla de TMT (todo menos tesis) y de TMI (todo menos investigación). La autora define al TMT en los siguientes términos:

Este síndrome se manifiesta objetivamente en los estudiantes por el abandono o postergación persistente en la elaboración del trabajo de investigación en cualquiera de sus etapas (generación, implementación, escritura, publicación), habiendo cumplido con los otros requisitos (...). (p. 34)

Sin embargo, la autora avanza y pone luz sobre una dificultad aún mayor, es decir, promueve conocimiento sobre el hecho de que TMT es uno de los aspectos que resultan de un paraguas de dificultades mayores, tal como las TMI. Conceptualiza estas de la siguiente manera: 
URL: http://www.una.ac.cr/educare

CORREO: educare@una.cr

Es el concurso de trastornos, impedimentos, obstáculos, actitudes, sentimientos y conductas inadecuadas, ausencia o deficiencia de algunas destrezas o lagunas de conocimiento, que presentan o enfrentan los estudiantes, profesores y profesionales cuando abordan la tarea de diseñar, planificar, desarrollar, escribir, supervisar y publicar trabajos de investigación o proyectos a largo plazo (...). (p. 255)

Si bien las dificultades puestas al desnudo a la hora de la elaboración y terminación de las tesinas son de carácter multifactorial, Rosas et al. $(2006$, p. 2) "(...) ha detectado que la influencia del Tutor es muy relevante para determinar el éxito en la culminación del trabajo (...)".

Entonces, teniendo en cuenta esta observación, sería interesante partir desde una clara conceptualización de tutor, para lo cual se han consultado múltiples fuentes.

Desde una perspectiva epistemológica, la palabra tutor viene del latín tutor, tutoris, cuyo significado es: persona que ejerce la tutela.

Según Ruiz (2002, p. 2), “(...) por tutela se entiende al ejercicio de la dirección, amparo o defensa de una persona respecto de otra".

De acuerdo con el Diccionario de la lengua española (Real Academia Española, 1992, p. 1448), “(...) el tutor es la persona encargada de orientar a los alumnos de un curso o de una asignatura. La misma fuente señala que la acción de la tutoría es un método de enseñanza por medio del cual un estudiante o un grupo pequeño de estudiantes reciben educación personalizada e individualizada de parte de un profesor".

La Enciclopedia internacional de la educación (Husén y Postlethwaite, 1992, p. 5729) sostiene que la tutoría se utiliza, principalmente, para proporcionar enseñanza compensatoria o complementaria a los estudiantes que tengan dificultades para aprender mediante los métodos convencionales o que tienen necesidades especiales que les impiden participar en un programa de enseñanza regular. La tutoría se lleva a cabo comúnmente, durante o después de la jornada escolar ordinaria y por obra de alguien que no es el maestro regular de las estudiantes o de los estudiantes.

La tutoría se considera también una forma “(...) de atención educativa donde el profesor apoya a un estudiante o a un grupo pequeño de estudiantes de una manera sistemática, [por medio de la] estructuración de objetivos, programas, organización por áreas, técnicas de enseñanza apropiadas [e] integración de grupos conforme a ciertos criterios y mecanismos de monitoreo y control, entre otros (...)" (Alcántara, 1990, p. 57).

Por otra parte, Latapí (1988) indica que es importante subrayar que la tutoría propicia una relación pedagógica diferente a la propuesta por la docencia cuando se ejerce ante grupos numerosos. En ese caso, el profesorado asume el papel de consejería o de compañero o compañera mayor. Ahí, el ambiente es mucho más relajado y amigable. Además, las condiciones del espacio físico, en donde tiene lugar la relación pedagógica, deben ser más acogedoras. 
Entonces, es aquí donde debe retomarse parte de la naturaleza del trabajo de tesina y no debe perderse de vista que se trata de trabajos dirigidos a un tribunal de especialistas o, por lo menos, de personas que ya han superado, en algún momento, la instancia de elaboración de un trabajo de este tipo. Además, son documentos con un formato particular, que Támola (2005) destaca como "(...) escritos, dirigidos por un director que debe orientar al tesista y hacerse cargo de la evaluación del contenido y de la forma de la tesina, será el director el que debe aprobar el borrador del trabajo final escrito a los efectos de su impresión definitiva" (p. 235).

Por tanto, es importante asimilar dos conceptos que denotan una misma función como son el de tutor y el de director, en tanto sintetizan la acción de determinados sujetos académicos que acompañan el quehacer de estudiantes en cuanto a la elaboración de su trabajo final.

En este sentido, se debe considerar que en el tutor se personifica alguien con experiencia en investigación y conocimientos sobre determinado campo disciplinar (Garritz y López, 1989); pero también es preciso tener en cuenta otro aspectos, como la disponibilidad de tiempo y la inclinación a colaborar con otros individuos sometidos a las exigencias de niveles de grado y posgrado.

Dada la relevancia y el alto nivel de implicancia en el desarrollo de la tarea de elaboración de la tesis, se retoma un concepto de Valarino (1997), citado por Rosas et al. (2006), quien define la tutoría o dirección de tesis de la siguiente manera:

(...) El profesor que ejerce el rol de supervisor académico de la tesis, asignado por las autoridades del programa de acuerdo con el estudiante, quien lo acompaña durante todo el proceso de investigación. Este rol está pobremente descrito en los documentos oficiales y se lo denomina de diversas maneras: profesor guía, profesor asesor, profesor consejero y tutor. (p. 129)

Ahora bien, sería interesante avanzar en el camino que orienta sobre las competencias propias de quien ejerce las labores de tutoría, dado que quienes solicitan su guía suelen realizarles demandas que trasuntan su función o responsabilidad.

En otros casos, muy actuales por cierto, el rol del tutor cumple una función meramente decorativa, en la medida que se solicita su presencia para cumplir los aspectos burocráticos institucionales, pero se hace evidente su ausencia al momento de asumir un compromiso concreto de acompañamiento del proceso.

A la figura del tutor o director, sería interesante sumar la figura del codirector o codirectora, que también se constituye en una figura de acompañamiento de la tarea y que, en ciertos casos, se vincula a la persona tesista por afinidad temática o cercanía jurisdiccional. 
URL: http://www.una.ac.cr/educare

CORREO: educare@una.cr

En fin, de la misma manera en que puede haber directores presentes o ausentes, puede haber codirectores en igualdad de condiciones. Por tanto, las garantías de la función no descansan en la presencia o la figuración.

La tarea de tutorear es una más en el amplio abanico del proceso de enseñanza aprendizaje y, en este sentido, Garritz y López (1989) plantean que la tutoría es un tipo de enseñanza personalizada, donde tutor y alumno son partícipes en un proceso de creación, en el cual, por lo general el estudiante aprende a realizar investigaciones. Entonces, como en toda instancia de este tipo, será función del tutor orientar, alentar y estimular al estudiante a fin de que este despliegue todas sus potencialidades.

El director o directora, por su parte, no se enfrenta a una tarea más, sino que se compromete en la realización de una serie de quehaceres que apelan a su formación de manera integral, tomando a su cargo demandas no solo referidas a "lo que sabe" sino que además interpelan "lo que es".

Respecto a las responsabilidades del tutor, diversos teóricos han realizado señalamientos.

Un buen modelo de responsabilidades, aún vigente, lo presenta la Universidad Autónoma de México (2009), donde se establecen, para el caso del posgrado concretamente de maestrías, determinadas responsabilidades que no escapan a ningún nivel de esta tarea, a saber:

- Supervisar de cerca el desarrollo de la investigación y la escritura de los resultados.

- Asesorar al estudiantado en la elección de seminarios y otras actividades académicas que contribuyan a su formación.

- Emitir una opinión cuando juzgue que ha sido adecuadamente concluida la escritura del trabajo.

- Informar por escrito al comité académico, sobre el desempeño de cada estudiante, por lo menos una vez por semestre.

- Asesorar al comité académico en los asuntos relacionados con los estudiantes y las estudiantes a su cargo.

Además de cursar en el cuarto semestre un seminario de investigación, a cargo de su director de trabajo final de investigación, el estudiante deberá participar en otras actividades de investigación afines a su trabajo y también coordinadas por su director. En caso de problemas en la relación estudiante-director y, si lo juzgase necesario, el comité académico, podrá [designar otra persona para la dirección]. (Universidad Nacional Autónoma de México [UNAM], 2009, Funciones del tutor principal de tesis, párr. 10)

Se puede agregar a estas responsabilidades y funciones previstas por la UNAM, algunas que Rosas et al. (2006) retoman de Mi Kim (1986), quien alude a aportes de Welsh (1978) y Moses (1984), donde expresamente mencionan entre las responsabilidades del director o tutor: 
Conocer con profundidad el área de estudio para poder proporcionar consejo, guía y crítica constructiva, ser capaz de establecer una relación interpersonal efectiva con sus tutoriados, para poder motivarles y ser un gerente eficaz que esté en capacidad de supervisar efectivamente la organización y ejecución del trabajo de grado. (p. 8).

No obstante lo mencionado, desde la perspectiva de otros textos consultados, sería importante incorporar a las funciones y responsabilidades, la necesidad de disponer de tiempo suficiente para realizar la tarea, contar con experiencia en trabajos de investigación y tener dominio de metodología de la investigación, en este último aspecto se concentrará la adecuada formulación del problema, los objetivos y la coherencia interna del estudio (González, 1993, citado en Rosas et al, 2006).

Si bien se trabaja con el rol de tutoría, es posible establecer que su protagonismo puede facilitar u obstaculizar la concreción de la tarea en cuestión, por lo que debería considerarse lo siguiente:

Entre las dificultades que se suelen vislumbrar, una se halla íntimamente asociada a la formación de la persona del director, dado que no siempre es posible encontrar en la misma persona toda la integralidad mencionada en la cita precedente. Ciertamente, una de las fallas más frecuentes se vincula al hecho de que en reiteradas ocasiones los estudiantes encuentran al Director que posee un cúmulo importante de conocimientos temáticos, vastos, resultante de años de estudio pero que, puede no tener la necesaria disposición a asumir la tarea de dirección por problemas de tiempo, de otros compromisos asumidos o de falta, incluso, de respaldo institucional. Por su parte, no siempre la formación disciplinar se halla complementada con la formación instrumental y de carácter transversal, como son, por ejemplo, el instrumental metodológico y a veces, estadístico. (Torres, Izura, Böhm, Lucero y Terrera 2011, p. 38)

Otro modelo interesante de caracterización de los directores, es el que surge de una investigación realizada por Rosas et al. (2006) y que presenta los siguientes resultados de acuerdo con la indagación realizada a una muestra determinada de estudiantes. Entre las características para el desempeño de sus funciones, se precisan las que se encontraron más relevantes:

- Mostrar experiencia investigativa.

- Demostrar seguridad en sus habilidades de asesoría e investigación.

- Ser responsables en el cumplimiento de sus funciones.

- Mostrar apertura a nuevas formas de abordar puntos sometidos a discusión.

- Demostrar experiencia para supervisar investigaciones. 
URL: http://www.una.ac.cr/educare

CORREO: educare@una.cr

- Tener estabilidad emocional.

- Aportar ideas y sugerencias constructivas.

- Facilitar información actualizada sobre el tema y las fuentes de información.

- Evidenciar destrezas en el manejo de información.

- Suministrar información al estudiante sobre líneas de investigación factibles.

Con respecto a las competencias:

- Mostrar experiencia investigativa.

- Demostrar seguridad en sus habilidades de asesoría e investigación.

- Ser responsables en el cumplimiento de sus funciones.

- Mostrar apertura a nuevas formas de abordar puntos sometidos a discusión.

- Demostrar experiencia para supervisar investigaciones.

Con respecto a las condiciones personales:

- Tener estabilidad emocional.

Con respecto a las funciones del rol:

- Aportar ideas y sugerencias constructivas.

- Facilitar información actualizada sobre el tema y las fuentes de información.

- Evidenciar destrezas en el manejo de información.

- Suministrar información al estudiante sobre líneas de investigación factibles.

Luego del recorrido bibliográfico y, muy particularmente, a partir del trabajo de campo realizado en la investigación marco de este escrito, es posible contribuir con algunos otros ítems que podrían considerarse, tales como la necesidad de que el profesor o profesora que va a desempeñar cargos de dirección de tesis cuente con un apoyo institucional que respalde su tarea, y que reconozca la carga que implica el seguimiento y acompañamiento en la elaboración de un trabajo de esta envergadura. 
Por otra parte, y apelando a los aportes disciplinares de la pedagogía, debe suplirse a quienes estén dispuestos a asumir estos compromisos de dirección, de todas las estrategias necesarias y contributivas para agilizar la realización del trabajo, como elementos favorecedores tanto de la tarea docente como de la receptividad estudiantil.

Asimismo, la actualización permanente constituye un requisito indispensable para lograr un acercamiento del director con sus estudiantes; además, actualmente resulta indispensable el uso y manejo de herramientas informáticas, de comunicación entre personas y de búsqueda de información. En el mismo sentido, el acceso y manejo de idiomas también deviene en un elemento favorecedor.

Por último (no todo se agota en aspectos externos o que puedan aprehenderse desde lo contextual), existen ciertos elementos endógenos como el rapport que pueda establecerse con los estudiantes, la paciencia, la disponibilidad, la pasión, los modos de enseñanza, las estrategias de transmisión de conocimientos $y$, por sobre todas las cosas, la transmisión de real interés por avanzar en las instancias de investigación, fundamentales frente a la dificultad evidenciada de baja terminalidad de estudios de grado.

El rol del la persona tutora es determinante a la hora de elaborar tesinas, y esto puede adquirir un cariz positivo o negativo, por lo que, en el caso de estudio, no puede dejar de considerarse como un aspecto de relevancia omnipresente en las causas por considerar cuando se analiza la dilación en los plazos de concreción de los estudios.

\section{Conclusiones}

En síntesis, se sabe que en la línea de las dificultades, el rol que pueda desempeñar el director o directora de tesis no es inocuo a los resultados que pueda alcanzar o no el estudiante.

Ciertamente, no se conoce el grado de incidencia que este rol puede tener en relación a la no terminación de la tesina o a la no incursión en la investigación, pero no puede dejar de considerarse como un factor de influencia.

Se evidencia, a través del trabajo bibliográfico, un interés especial por parte de las instituciones en destacar el papel fundamental del tutor o director, al poner especial énfasis en sus funciones y responsabilidades. Por tanto, es de interés institucional su existencia y la delimitación de los alcances de su incumbencia.

Sin duda, la cercanía a la etapa de culminación de la carrera significa, para muchos estudiantes, el inicio de un largo camino que, impregnado de soledad y fuera de ritmo en relación con las prácticas que se venían desarrollando, se convierte en un desafío que rompe con la inercia que se puede haber tenido a lo largo de la época de cursado y de presentación de materias. 
URL: http://www.una.ac.cr/educare

CORREO: educare@una.cr

Sucede, también, que en muchos casos los estudiantes depositan demasiadas expectativas en el tutor o tutora y pierden de vista que "es solo eso" y que, de una u otra forma, también corresponde a la función de dirección otorgar márgenes de soledad y libertad para que el estudiantado se vea forzado a superar escollos y a aprender esta nueva forma de ganar conocimientos.

Si bien se han señalado diversas apreciaciones sobre las funciones y responsabilidades esperables de un director o directora, debe insistirse en el hecho de que esta figura no es garante del éxito en la realización de la tarea.

Entonces, la función de acompañamiento, que tan bien se ha descripto desde los aportes de los especialistas mencionados, no debe ser intrusiva, sino guardar la distancia precisa para estimular la acción de trabajo del estudiante; acompañarle, pero no realizar las tareas que garantizan el aprendizaje de, por ejemplo, la aplicación de los procesos de investigación.

Tampoco hay que desestimar otros aspectos externos a la persona directora, y más vinculados a cuestiones de índole institucional, como son la ausencia de apoyo para el desempeño del rol, la carencia de estímulos para llevar a cabo esta tarea e incluso falencias en programas de capacitación y actualización permanentes, pues estos últimos pudieran ser favorecedores de estrategias de vinculación con los estudiantes, que por lo general pertenecen a grupos generacionales alternativos al de los directores.

Surge aquí algo interesante que tiene que ver con una serie de cuestionamientos vinculados a si el director o tutor es alguien imprescindible para la labor institucional que garantiza la culminación de una carrera de grado, y hasta qué punto debería la institución en cuestión, estimular, favorecer y valorar la tarea que desempeña.

¿No debería la propia institución vehiculizar los mecanismos imprescindibles para que los profesionales puedan desempeñar el rol de dirección, dado que estas no son competencias incluidas en la formación de grado de los propios docentes, sino que exigen de formación, de tiempo, de investigación, de estudio y de trabajo, extraordinarios a sus tareas habituales?

Ciertamente, la elaboración de las tesis, tal como se presumía al momento de elaborar este trabajo, evidencia dificultades. Probablemente la institución deberá reparar en los incidentes que emergen en esta etapa y que generan profundas brechas en la mayoría de los casos.

Las demoras que se evidencian para la culminación de los estudios, ¿pueden relacionarse con un compromiso institucional o la ausencia de él, en el que de alguna manera se acuerde con las personas directoras para ese acompañamiento final, en el sentido de que no debe exceder la cuantía de años que aquí se han presentado?

Otro aspecto particular es el número de mujeres abocadas al estudio de las diversas carreras, lo que coincide con otros datos de la realidad que refieren al sector de educación como preferente y mayoritariamente desempeñado por mujeres. Por supuesto, existen algunas excepciones que en este caso tienen que ver con dos carreras, Licenciatura en Educación Ambiental y en Documentación y Gestión de la Información. 
También se puede resaltar que, al tomar el caudal de alumnos que egresaron en los años 2010 y 2011, esto permite observar el flujo de egresados por carrera, donde se pone de manifiesto la masividad de algunas especialidades frente a pequeños números de egresados en otras.

En relación con la hipótesis formulada para este estudio, que sostenía que: la terminación de las tesinas en las diversas carreras de licenciatura de la Facultad de Educación Elemental y Especial, presenta importantes brechas temporales, años de desgranamiento entre la culminación del tramo de aprobación de la última materia y la finalización de dicha tesina, es posible, desde la evidencia empírica que proporciona la información en cifras, verificarla, dado que se constituye en el tópico central la mencionada brecha entre los dos momentos señalados.

Sin embargo, no se puede dejar de destacar que los promedios de las calificaciones obtenidas en la presentación y defensa de la tesina evidencian altas notas, con lo que no se ponen de manifiestos problemas estructurales vinculados a la elaboración de los conocimientos y los saberes. Por tanto, ¿hasta qué punto puede vincularse la demora en la realización a una suerte de desafección institucional que se ha motivado por falta de contención, falta de docentes disponibles para la dirección, falta de acompañamiento efectivo, falta de compromiso que implique el cumplimiento de plazos, más allá de las naturales dificultades particulares que pueda tener una persona frente a la tarea de elaborar una tesis?

En fin, hasta aquí se han expuesto solo los aspectos sobresalientes, podría continuarse con la elaboración de nuevos cruces de variables y la profundización de categorías de análisis que generaran más información; no obstante, esta es vasta, en el sentido de los objetivos previstos para este escrito.

\section{Referencias}

Alcántara, A. (julio-diciembre, 1990). Consideraciones sobre la tutoría en la docencia universitaria. Perfiles Educativos, 49-50, 51-58. Recuperado de http://www.iisue.unam.mx/perfiles/ index.php?numero $=49-50 \&$ anio $=1990$

Borsinger, A. (2005). La tesis. En: L. Cubo (ed.), Los textos de la ciencia (pp. 267- 282). Córdoba: Comunicarte.

Garritz, A. y López, D. M. (marzo, 1989). Tutoría: El perfil del docente en el postgrado. I/ Seminario Latinoamericano de Estudios de Postgrado. Seminario llevado a cabo en Universidad de Lima, Lima, Perú.

Husén, T. y Postlethwaite, T. N. (eds.). (1992). Enciclopedia internacional de educación (Vol. 9, pp. 5729-5732), España: Ministerio de Educación y Ciencia.

Latapí, P. (octubre-diciembre, 1988). La enseñanza tutorial: Elementos para una propuesta orientada a elevar la calidad. Revista de la Educación Superior, 68, 5-19. 
URL: http://www.una.ac.cr/educare

CORREO: educare@una.cr

Real Academia Española (1992). Diccionario de la lengua española. Madrid: Real Academia Española.

Rosas, A. K., Flores, D. y Valarino, E. (junio, 2006). Rol del tutor de tesis: Competencias, condiciones personales y funciones. Investigación y Postgrado, 21(1), 1-24.

Ruiz, C. (2002). Cómo llegar a ser un tutor competente. Caracas: Santillana.

Támola, D. (2005). La tesina de licenciatura. En L. Cubo (ed.), Los textos de la ciencia (pp. 235265). Córdoba: Comunicarte.

Torres, L. M., Izura, T. A., Böhm, F. M., Lucero, A. E. y Terrera, S. E. (2011). Descripción y análisis de las mayores dificultades que visualizan y narran, alumnos y docentes, a lo largo del proceso de elaboración de las tesis de licenciatura, Facultad de Educación Elemental y Especial, Universidad Nacional de Cuyo (Informe final de investigación). Universidad del Cuyo, Argentina.

Universidad Nacional Auónoma de México [UNAM]. (2009). Responsabilidades y funciones de los tutores. México: Posgrado en Filosofía de la Ciencia, UNAM. Recuperado de http://www. posgrado.unam.mx/filosofiadelaciencia/tutores-resp.html

Universidad Nacional de Cuyo (UNCUYO). Facultad de Educación Elemental y Especial (FEEyE). (2011). Secretaria Académica. Argentina: Autor.

Universidad Nacional de Cuyo (UNCUYO). Facultad de Educación Elemental y Especial (FEEyE). (s. f.). Presentación. Recuperado de http://www.educacion.uncu.edu.ar/paginas/index/ presentacion

Valarino, E. (1997). Tesis a tiempo (2a ed.). Barcelona, España: Grupo Editorial Carnero.

\section{Cómo citar este artículo en APA:}

Alcia, I., Torres, L. M., Böhm, F. M., Terrera, S. E. y Lucero, A. E. (2013). La elaboración de la tesina como la última cuesta en la graduación: Sus dificultades y el rol de tutoría. Revista Electrónica Educare, 17(2), 123-144. Recuperado de http://www.revistas.una. ac.cr/index.php/EDUCARE/issue/current

Nota: Para citar este artículo en otros sistemas puede consultar el hipervínculo "Como citar el artículo" en la barra derecha de nuestro sitio web:

http://www.revistas.una.ac.cr/index.php/EDUCARE/index 